\title{
Random regression models using Legendre polynomials or linear splines for test-day milk yield of dairy Gyr (Bos indicus) cattle
}

\author{
R. J. Pereira, ${ }^{* 1}$ A. B. Bignardi, ${ }^{*}$ L. El Faro,† R. S. Verneque, $\ddagger$ A. E. Vercesi Filho, $†$ and L. G. Albuquerque ${ }^{*}$ \\ ${ }^{*}$ Faculdade de Ciências Agrárias e Veterinárias, Universidade Estadual Paulista Júlio de Mesquita Filho, CEP 14884-900, Jaboticabal, SP, Brazil \\ †Agência Paulista de Tecnologia dos Agronegócios-APTA, CEP 14030-670, Ribeirão Preto, SP, Brazil \\ ‡Embrapa Gado de Leite, CEP 36038-330, Juiz de Fora, MG, Brazil
}

\section{ABSTRACT}

Studies investigating the use of random regression models for genetic evaluation of milk production in Zebu cattle are scarce. In this study, 59,744 test-day milk yield records from 7,810 first lactations of purebred dairy Gyr (Bos indicus) and crossbred (dairy Gyr $\times$ Holstein) cows were used to compare random regression models in which additive genetic and permanent environmental effects were modeled using orthogonal Legendre polynomials or linear spline functions. Residual variances were modeled considering 1,5 , or 10 classes of days in milk. Five classes fitted the changes in residual variances over the lactation adequately and were used for model comparison. The model that fitted linear spline functions with 6 knots provided the lowest sum of residual variances across lactation. On the other hand, according to the deviance information criterion (DIC) and Bayesian information criterion (BIC), a model using third-order and fourth-order Legendre polynomials for additive genetic and permanent environmental effects, respectively, provided the best fit. However, the high rank correlation (0.998) between this model and that applying third-order Legendre polynomials for additive genetic and permanent environmental effects, indicates that, in practice, the same bulls would be selected by both models. The last model, which is less parameterized, is a parsimonious option for fitting dairy Gyr breed test-day milk yield records.

Key words: random regression model, covariance function, orthogonal polynomial, spline

\section{INTRODUCTION}

In tropical regions, dairy farmers mainly use pasturebased production systems. In these systems, in which the animals are frequently exposed to climatic varia-

Received October 15, 2011.

Accepted September 6, 2012.

${ }^{1}$ Corresponding author: rodjunper@gmail.com tions and to endo- and ectoparasites, European breeds show problems of adaptation that interfere with the productive and reproductive performance and health of the animals. As a consequence, in view of its adaptation to these environments, the dairy Gyr (Bos indicus) breed is present in more than $80 \%$ of Brazilian dairy herds as purebreds or as crossbreeds with Holstein cattle (Reis Filho, 2006). In general, genetic breeding programs for dairy Zebu breeds use 305-d milk yield estimated from test-day records obtained across lactation as a selection criterion. Genetic evaluations for milk yield for these breeds are performed under an animal model based on 305-d first-lactation yields (Verneque et al., 2011). Today, test-day models are much more common than lactation models for genetic evaluations of production traits worldwide (Interbull, 2012). Some benefits attributed to the adoption of test-day models are better definition of contemporary groups and removal of environmental effects and, therefore, more accurate genetic evaluations of cows and bulls (Schaeffer et al., 2000). Also, it allows the inclusion of incomplete lactation data without the need for projections, thus allowing more frequent evaluations and reducing generation intervals (Swalve, 2000). Among the genetic evaluation models using test-day records, those applying random regressions, in general, are more parsimonious than multivariate models that consider production in each of the 10 mo of lactation as a different trait.

Orthogonal Legendre polynomials have been frequently used to model the covariance structure between test-day records by random regression models (RRM; Bohmanova et al., 2008; El Faro et al., 2008; Bignardi et al., 2009). According to Meyer (2005), changes in variance along a continuous scale are generally well modeled by high-order Legendre polynomials, but these polynomials may provide implausible parameter estimates for the beginning and the end of the trajectory by overemphasizing observations at the extremes. One alternative to high-order Legendre polynomials are segmented polynomials (splines). Splines are defined as curves that consist of individual segments of lower-degree polynomials joined at specific points, called knots 
(Meyer, 2005). According to Misztal (2006), each coefficient of a spline function affects only a fraction of the trajectory studied, resulting in possibly better numerical properties and facilitating the process of parameter estimation. In addition, the positions of the knots can be chosen according to the pattern of changes along the trajectory, with knots being positioned denser in regions of fast changes and more distant in other regions.

Studies investigating the use of RRM for the genetic evaluation of milk production in Zebu breeds are scarce. Costa et al. (2005), using milk yield records of a small sample (1,200 cows) of dairy Gyr, compared RRM applying various functions (Wilmink or Ali and Schaeffer) or Legendre polynomials. The authors found very high (around 0.80 ) estimates of heritability at the first $60 \mathrm{~d}$ of lactation and negative genetic correlations estimates between days at the beginning and at the end of lactation. Herrera et al. (2008) and Pereira et al. (2010), working with larger samples and grouping test-day records into ten 30 -d or twenty 15 -d classes, respectively, compared Wilmink to Ali and Schaeffer functions and concluded that the latter function fitted the data best. Heritability estimates obtained in both works were similar and ranged from 0.09 to 0.33 . However, Pereira et al. (2010) reported negative genetic correlations between test-day records at the beginning and at the end of lactation. None of these studies used spline functions or crossbred animals. It is important to point out that the current dairy Gyr genetic evaluation includes milk yield records from purebred and crossbred animals. In this respect, the choice of an adequate model will allow the application of RRM to genetic evaluations of dairy Zebu breeds. Therefore, the objective of the present study was to compare various RRM for fitting test-day milk yield records of first-lactation dairy Gyr cows using orthogonal Legendre polynomials or linear spline functions of DIM.

\section{MATERIALS AND METHODS}

\section{Data}

For the estimation of variance components, the data set contained 59,744 test-day records from 7,810 first lactations of purebred dairy Gyr and crossbred (dairy Gyr $\times$ Holstein) cows, daughters of 765 dairy Gyr sires, calved between 1990 and 2009 and belonging to 229 herds. Age at calving ranged from 24 to 60 mo. The data were obtained from the Brazilian National Dairy Cattle Archive. All records were originated from supervised testing and test-day intervals were between 15 and $45 \mathrm{~d}$. Only test-day records obtained between 5 and 305 DIM were used. The criteria for inclusion of the cows in the study were first test-day record obtained within $45 \mathrm{~d}$ after calving and the number of test-day records higher than 3. Proportions of cows according to the number of test-day records were as follows: 4 records $=3.6 \%, 5$ records $=5.6 \%, 6$ records $=8.3 \%, 7$ to 10 records $=81.1 \%$, and $>10$ records $=1.4 \%$. The contemporary groups were formed by animals whose test day was measured in the same herd, year, and month. Contemporary groups containing fewer than 4 animals were eliminated. The cows were divided into 5 genetic groups based on percentage of dairy Gyr bloodline (DG): $1=100 \%, 2=87.5 \% \leq \mathrm{DG}<100 \%, 3=75 \%$ $\leq \mathrm{DG}<87.5 \%, 4=62.5 \% \leq \mathrm{DG}<75 \%$, and $5=50 \%$ $\leq \mathrm{DG}<62.5 \%$. About $80 \%$ of the cows were in genetic groups 1 and 4 . Descriptive statistics are presented in Table 1 . The pedigree was traced back 5 generations, resulting in a pedigree file containing 16,546 animals.

\section{Models}

Analysis was performed using RRM. The systematic effects of contemporary group, linear and quadratic effects of age of cow at calving, and the average lactation curve for each genetic group (fixed regression), modeled using a third-order Legendre polynomial, were included. Additive genetic and permanent environmental effects were modeled using orthogonal Legendre polynomials or linear spline functions.

The general RRM used in the analyses can be written as

$$
\begin{aligned}
& y_{i j k l}=C G_{i}+\sum_{n=1}^{2} b_{n} x_{k}^{n}+\sum_{m=1}^{4} \beta_{j m} \varphi_{m}(t) \\
& +\sum_{m=1}^{c} \alpha_{k m} \varphi_{m}(t)+\sum_{m=1}^{c} p_{k m} \varphi_{m}(t)+e_{i j k l},
\end{aligned}
$$

where $y_{i j k l}$ is the lth observation recorded on day of lactation $t$ in animal $k$, which belongs to the genetic group $j$ and contemporary group $i$; $C G_{i}$ is the effect of the $i$ th contemporary group (5,747 classes); $b_{n}$ is the regression coefficient for the linear $(\mathrm{n}=1)$ and quadratic $(\mathrm{n}=$ 2) effects of cow's age $x_{k}$ at calving, in months; $\beta_{j m}$ is the set of $m$ fixed regression coefficients for the mean trajectory of genetic group $j ; \varphi_{m}(t)$ is the covariate of the regression function according to day of lactation $(t) ; \alpha_{k m}$ and $p_{k m}$ are sets of $m$ regression coefficients for additive genetic and permanent environmental effects of each cow $k$; and $e_{i j k l}$ is the temporary measurement error associated with observation $l$ of cow $k$, which belongs to contemporary group $i$ and genetic group $j$. The number of regression coefficients $c$ varies according to the functions used for additive genetic and permanent environmental random regressions. 
Table 1. Descriptive statistics of the data by genetic group

\begin{tabular}{|c|c|c|c|c|c|}
\hline Item & \multicolumn{5}{|c|}{ Genetic group $^{1}$} \\
\hline Number of records & 36,874 & 2,252 & 5,538 & 12,594 & 2,485 \\
\hline Average peak yield (kg) & 12.17 & 9.91 & 10.51 & 11.05 & 13.56 \\
\hline Average DIM at peak (d) & 31 & 24 & 32 & 29 & 21 \\
\hline Average lactation length (d) & 302 & 301 & 304 & 302 & 307 \\
\hline
\end{tabular}

${ }^{1}$ Genetic groups: $1=100 \%$ dairy Gyr (DG), $2=87.5 \% \leq \mathrm{DG}<100 \%, 3=75 \% \leq \mathrm{DG}<87.5 \%, 4=62.5 \% \leq \mathrm{DG}<75 \%$, and $5=50 \% \leq$ DG $<62.5 \%$.

Residual variance was assumed to be (a) constant (homogeneous) across lactation or (b) heterogeneous, considering 5 classes (5-30, 31-60, 61-120, 121-270, and 271-305 DIM), or 10 classes (5-30, 31-60, 61-90, 91-120, 121-150, 151-180, 181-210, 211-240, 241-270, and 270-305 DIM). When 5 classes were used, the variances were divided according to similarity based on the residual variance estimated with the model containing 10 classes. When evaluating the different residual variance structures, the random regressions for the additive genetic and permanent environmental effects were modeled using third-order Legendre polynomials.

After the best residual variance structure was chosen, based on DIC and BIC criteria, different RRM were applied to analyze the data. Additive genetic and permanent environmental effects were modeled using orthogonal Legendre polynomials of order 3, 4, or 5, and linear spline functions with 4 to 6 knots. For spline functions, the knots were first positioned at 5, 29, 71, and 305 DIM. The first internal knot was meant to be around the lactation peak (Table 1) and, in this region, the day with the largest number of records was the 29th. To keep shorter intervals between knots at the beginning of the lactation, the second knot was placed on $d$ 71. Considering the occurrence of lactations shorter than $200 \mathrm{~d}$, usual for DG, 2 additional knots were placed at d 120 and 260, to increase flexibility. When linear spline functions were used, additive genetic and permanent environmental random effects were modeled using the same number of segments for each model studied.

The different models are presented according to the code Tkakpr, where $T$ is the type of basis function used (orthogonal Legendre polynomial, LEG, or linear spline function, SPL), $k a$ and $k p$ are the numbers of random regression coefficients for additive genetic and permanent environmental effects, respectively, and $r$ is the number of residual variance classes.

The analyses were performed by Bayesian inference using the GIBBS3F90 program (Misztal, 2010). The RRM can be rewritten in matrix form as follows:

$$
\mathbf{y}=\mathbf{X b}+\mathbf{Z a}+\mathbf{W} \mathbf{p}+\mathbf{e}
$$

where $\mathbf{y}$ is the vector of observations; $\mathbf{b}$ is the vector of systematic effects; a is the vector of the random regression coefficients of additive genetic effects of the animal; $\mathbf{p}$ is the vector of random regression coefficients of permanent environmental effects; $\mathbf{e}$ is the vector of residual effects; and $\mathbf{X}, \mathbf{Z}$, and $\mathbf{W}$ are incidence matrices corresponding to the observations for systematic, additive genetic, and permanent environmental effects, respectively. It was assumed that

$$
\begin{gathered}
\mathbf{y} \mid \mathbf{b}, \mathbf{a}, \mathbf{p}, \sigma_{\mathrm{e}_{1}}^{2}, \ldots, \sigma_{\mathrm{e}_{\mathrm{x}}}^{2} \sim \operatorname{NMV}(\mathbf{X b}+\mathbf{Z a}+\mathbf{W} \mathbf{p}, \mathbf{R}), \\
\mathbf{R}=\operatorname{diag}\left\{\sigma_{e_{x}}^{2}\right\},
\end{gathered}
$$

with $x$ ranging from 1 to $n$, where $n$ is the number of residual classes.

The assumptions regarding prior distributions for unknown parameters were a $\mid \boldsymbol{\Lambda}_{\mathrm{a}} \sim \operatorname{NMV}(0, \mathbf{G})$, with $\mathbf{G}$ $=\boldsymbol{\Lambda}_{\mathrm{a}} \otimes \mathbf{A}$, where $\mathbf{A}$ is the numerator relationship matrix between animals and $\boldsymbol{\Lambda}_{\mathrm{a}}$ is the matrix containing covariances between regression coefficients for additive genetic effects; $\mathbf{p} \mid \boldsymbol{\Lambda}_{\mathbf{p}} \sim \operatorname{NMV}(0, \mathbf{P})$, with $\mathbf{P}=\boldsymbol{\Lambda}_{\mathrm{p}} \otimes \mathbf{I}$, where $\mathbf{I}$ is the identity matrix and $\boldsymbol{\Lambda}_{\mathrm{p}}$ is the matrix containing covariances between random regression coefficients for permanent environmental effects; $\Lambda_{\mathrm{a}}\left|\mathrm{v}_{\mathrm{a}}, \mathrm{S}_{\mathrm{a}}^{2} \sim \mathrm{W}^{-1}\left(\mathrm{v}_{\mathrm{a}}, \mathrm{v}_{\mathrm{a}} \mathrm{S}_{\mathrm{a}}^{2}\right), \quad \Lambda_{\mathrm{p}}\right| \mathrm{v}_{\mathrm{p}}, \mathrm{S}_{\mathrm{p}}^{2} \sim \mathrm{W}^{-1}\left(\mathrm{v}_{\mathrm{p}}, \mathrm{v}_{\mathrm{p}} \mathrm{S}_{\mathrm{p}}^{2}\right)$, and $\mathbf{R} \mid \mathrm{v}_{\mathrm{e}}, \mathrm{S}_{\mathrm{e}}^{2} \sim \mathrm{W}^{-1}\left(\mathrm{v}_{\mathrm{e}}, \mathrm{v}_{\mathrm{e}} \mathrm{S}_{\mathrm{e}}^{2}\right)$, where $\mathrm{v}_{\mathrm{a}}, \mathrm{S}_{\mathrm{a}}^{2}, \mathrm{v}_{\mathrm{p}}, \mathrm{S}_{\mathrm{p}}^{2}$, and $\mathrm{v}_{\mathrm{e}}, \mathrm{S}_{\mathrm{e}}^{2}$ correspond to the degree of belief and prior values for covariances of additive genetic and permanent environmental regression coefficients and for residual variances, respectively; and $\mathbf{b} \propto$ constant. Terms NMV and $\mathrm{W}^{-1}$ correspond to multivariate normal and inverse Wishart distributions, respectively.

The inferences on the parameters of interest were made based on their corresponding marginal posterior distributions. A chain of 850,000 samples was generated for each model studied. Using a conservative approach, the first 50,000 samples were discarded and were con- 
Table 2. Average, minimal (Min), and maximal (Max) number of independent samples for genetic (G), permanent environmental (PE), and residual covariance components in 16,000 samples after burn-in and thin

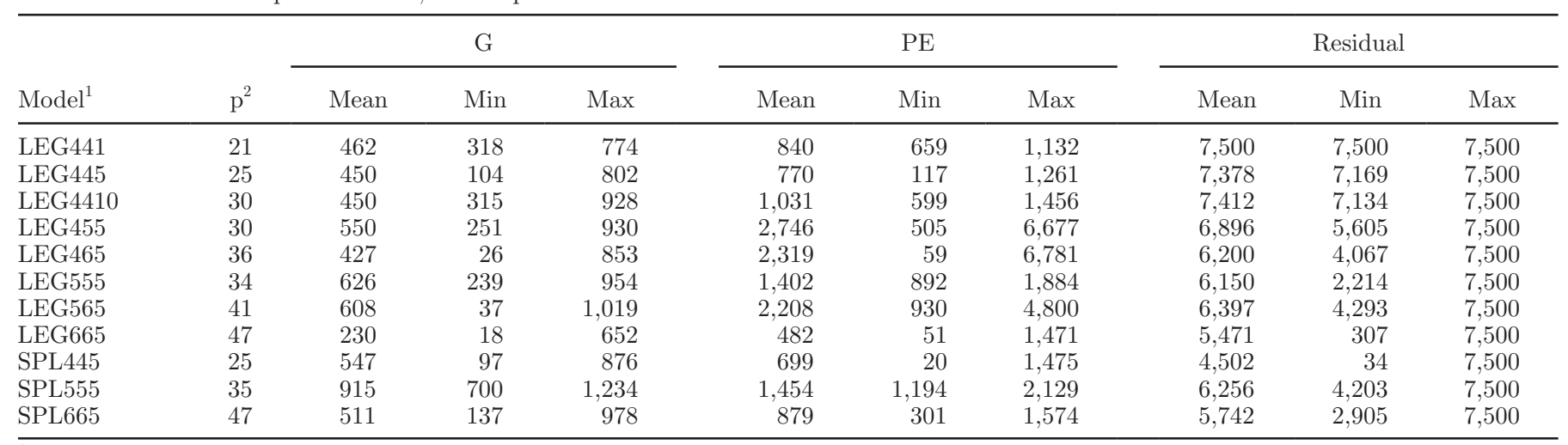

${ }^{1}$ Models are presented according to the code Tkakpr, where $T$ is the type of basis function used (orthogonal Legendre polynomial, LEG, or linear spline function, SPL); $k a$ and $k p$ are the numbers of random regression coefficients for additive genetic and permanent environmental effects, respectively; and $r$ is the number of residual variance classes.

${ }^{2}$ Number of parameters.

sidered as burn-in. Convergence was monitored by graphic inspection of samples $\times$ iterations. In addition, the criteria proposed by Heidelberger and Welch (1983) and by Geweke (1992) were used, which were calculated using the boa package (Smith, 2007) of the R program. Using a thin of 50, inferences were performed on the remaining 16,000 samples. Summary statistics of effective sample sizes are showed on Table 2.

For illustration, the genetic parameters for days of lactation were obtained at intervals of $15 \mathrm{~d}$ for each sample. Because linear spline functions are not orthogonal, the eigendecomposition of the covariance matrix between random regression coefficients does not produce the eigenvalues and eigenfunctions of the covariance function (Meyer, 2005). Therefore, as suggested by Rice and $\mathrm{Wu}$ (2001), the eigenvalues and eigenfunctions were obtained numerically by evaluating the covariance function every $15 \mathrm{~d}$ over an interval of 5 to $305 \mathrm{~d}$ of lactation and then calculating the eigenvalues and eigenfunctions of the resulting covariance matrix between the test-day records.

\section{Model Comparison}

The RRM were compared using the following criteria:

1. Total residual variance (TRV): $T R V_{m}=\sum_{d=5}^{305} r v_{m d}$, where $r v$ is the posterior mean of residual variance for day $d$.

2. Deviance information criterion (DIC) proposed by Spiegelhalter et al. (2002):

$\underline{D I C}=\bar{D}(\theta)+p_{D}=2 \bar{D}(\theta)-D(\bar{\theta})$,

where

$\bar{D}(\theta)=E_{\theta \mid y}[D(\theta)]$ (posterior expectation of the
Bayesian deviance) and $D(\theta)=-2 \log p(y \mid \theta)$, indicating the goodness of fit of the model. The effective number of parameters represents a penalty for increasing model complexity:

$p_{D}=\bar{D}(\theta)-D(\bar{\theta})$, where $\theta$ is the vector of model parameters and $D(\bar{\theta})$ is the Bayesian deviance evaluated at the posterior mean of the parameters.

3. Bayesian information criterion (BIC) proposed by Schwarz (1978):

$B I C=\bar{D}(\theta)+\operatorname{par}[\log (n)]$, where par is the number of parameters in the model and $\log (n)$ is the natural logarithm of the total number of observations.

Lower DIC and BIC are preferred, as they indicate a better goodness of fit associated with a lower degree of complexity. In addition, the 2 best models according to the DIC and BIC criteria were compared based on the percent relative reduction of complexity (PRRC) proposed by Bozdogan (2000):

$$
P R R C_{m}=\left[C_{1}\left(\boldsymbol{\Lambda}_{\mathrm{m}}\right)-C_{1}\left(\boldsymbol{\Lambda}_{\mathbf{R m}}\right)\right] / C_{1}\left(\boldsymbol{\Lambda}_{\mathrm{m}}\right),
$$

where $\boldsymbol{\Lambda}_{\mathrm{m}}$ and $\boldsymbol{\Lambda}_{\mathrm{Rm}}$ are covariance and correlation matrices between the parameters of model $m$, and $C_{1}\left(\boldsymbol{\Lambda}_{\mathrm{m}}\right)$ $=\operatorname{rank}\left(\boldsymbol{\Lambda}_{\mathrm{m}}\right) \log \left[\operatorname{trace}\left(\boldsymbol{\Lambda}_{\mathrm{m}}\right) / \operatorname{rank}\left(\boldsymbol{\Lambda}_{\mathrm{m}}\right)\right]-\log \left[\left|\boldsymbol{\Lambda}_{\mathrm{m}}\right|\right]$. A higher magnitude of PRRC indicates lower complexity of the model. Models were further compared by the rank of the animals according to their EBV for 305-d cumulative milk yield.

\section{RESULTS AND DISCUSSION}

The overall average test-day milk yield was $9.81 \mathrm{~kg}$, with a standard deviation of $4.39 \mathrm{~kg}$ and coefficient of 


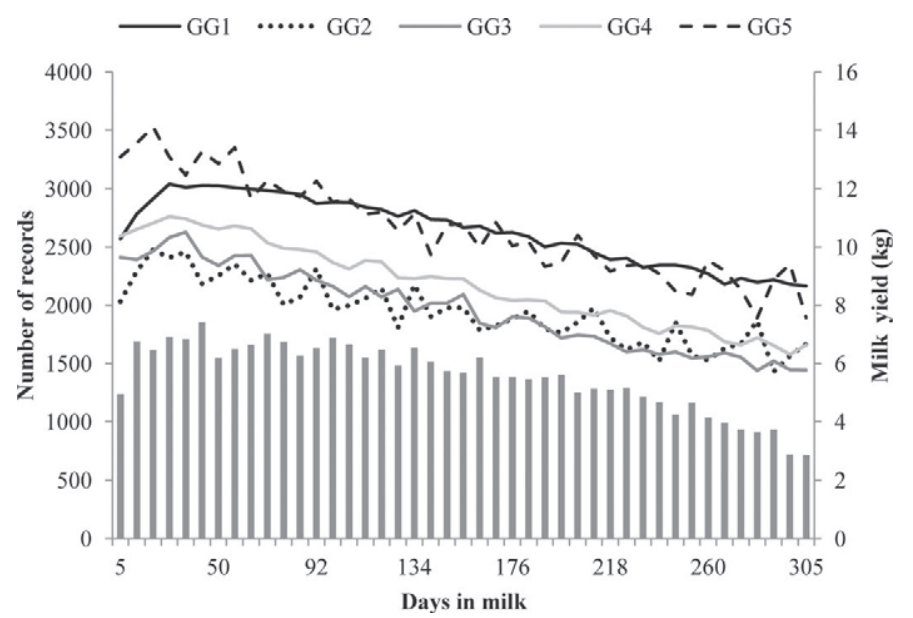

Figure 1. Number of records (bars) and average milk yield (lines by genetic group, GG) according to DIM (7-d classes). Genetic groups: GG1 $=100 \%$ dairy Gyr (DG), GG2 $=87.5 \% \leq \mathrm{DG}<100 \%, \mathrm{GG} 3=$ $75 \% \leq \mathrm{DG}<87.5 \%, \mathrm{GG} 4=62.5 \% \leq \mathrm{DG}<75 \%$, and $\mathrm{GG} 5=50 \%$ $\leq \mathrm{DG}<62.5 \%$.

variation of $44.8 \%$. An increase in milk production was observed up to $\mathrm{d} 30$ of lactation, followed by a gradual decrease until the end of lactation (Figure 1). Due to the differences in production level between genetic groups (Figure 1), it was important to consider specific fixed regressions for each genetic group.

The effective sample sizes (ESS) for the estimated covariance components varied across models and effects (Table 2). The ESS was substantially smaller for additive genetic and permanent environmental effects than for residual effects. In general, the pattern of differences in ESS of the covariance components did not indicate any clear association between model and the ESS. However, for models LEG465, LEG565, and LEG665, the minimum ESS was smaller than those for the other models, indicating that some Markov chains for the covariance components, mainly for the covariances between higher-order coefficients, may be affected by poor mixing due to complexity of these models (more parameterized).

The results of the different model comparison criteria are shown in Table 3 . When the residual variance structure was analyzed, all criteria indicated the need to consider heterogeneity of variances across lactation. Comparison of the models containing 5 (LEG445) and 10 (LEG4410) residual variance classes showed a lower total residual variance for model LEG4410. In contrast, model LEG445 presented lower DIC and BIC values, indicating a better fit provided by this model compared with LEG441 and LEG4410. The posterior mean of residual variance for the LEG441 was 1.35. On the other hand, residual variances for the LEG445 were $2.45,1.96$, $1.38,1.16$, and 0.82 for the 5 to 30,31 to 60,61 to 120 , 121 to 270 , and 271 to 305 DIM classes, respectively. This pattern is similar to that found by Pereira et al. (2010). However, Costa et al. (2005) found a different pattern, with higher estimates at the extremes of lactation when compared with the middle. These results demonstrate that residual variances are heterogeneous across lactation and that 5 classes seem to be sufficient to model the effect in this population. Similar results have been reported by Costa et al. (2005), Herrera et al. (2008), and Pereira et al. (2010) for the dairy Gyr breed and by Rekaya et al. (1999), López-Romero and Carabaño (2003), and Bignardi et al. (2009) for Holstein cattle.

The models using Legendre polynomials of orders higher than 3 for additive genetic effects and higher than 4 for permanent environmental effects provided negative genetic correlation estimates between test-day yields at the beginning and at the end of lactation and were, therefore, excluded. This behavior is not expected in biological terms and has been attributed to poor fit of RRM at the extreme points of the curve, probably because of the smaller number of observations during these periods (Meyer, 1999). Pereira et al. (2010), analyzing part of the same data set as used in the present

Table 3. Number of random regression coefficients for the additive genetic $\left(k_{a}\right)$ and permanent environmental $\left(k_{p}\right)$ effects, number of residual classes (r), position of knots (pk), number of parameters (p), total residual variance (TRV), and deviance (DIC) and Bayesian (BIC) information criteria

\begin{tabular}{|c|c|c|c|c|c|c|c|c|}
\hline Model $^{1}$ & $\mathrm{k}_{\mathrm{a}}$ & $\mathrm{k}_{\mathrm{p}}$ & $\mathrm{r}$ & $\mathrm{pk}$ & $\mathrm{p}$ & $\mathrm{TRV}^{2}$ & $\mathrm{DIC}^{2}$ & $\mathrm{BIC}^{2}$ \\
\hline LEG445 & 4 & 4 & 5 & - & 25 & 29.0 & 2,413 & 3,259 \\
\hline LEG4410 & 4 & 4 & 10 & - & 30 & 25.0 & 2,899 & 3,297 \\
\hline SPL445 & 4 & 4 & 5 & $5,29,71,305$ & 25 & 57.5 & 4,707 & 6,908 \\
\hline SPL555 & 5 & 5 & 5 & $5,29,71,120,305$ & 35 & 21.9 & 2,183 & 2,348 \\
\hline SPL665 & 6 & 6 & 5 & $5,29,71,120,260,305$ & 47 & 0 & 546 & 67 \\
\hline
\end{tabular}

${ }^{1}$ Models are presented according to the code Tkakpr, where $T$ is the type of basis function used (orthogonal Legendre polynomial, LEG, or linear spline function, SPL); $k a$ and $k p$ are the numbers of random regression coefficients for additive genetic and permanent environmental effects, respectively; and $r$ is the number of residual variance classes.

${ }^{2}$ Values scaled as deviation from the respective "best" values (0). 
study using Wilmink or Ali and Schaeffer functions to model fixed and random regressions, found negative genetic correlations between test-day milk yield records at the beginning and at the end of lactation. One another possible explanation reported by the authors is the application of bovine somatotropin together with preferential treatment to part of the animals in some herds. Because this practice is generally performed after the second month of lactation, cows receiving this treatment may present even higher milk yields at the end of lactation when compared with the beginning of lactation. No information is available in the database about the use of somatotropin and, as this effect was not included in the model, interferences with the genetic parameter estimates cannot be ruled out. Negative correlations between the extreme points of the lactation curve have been reported by Costa et al. (2005) for the dairy Gyr; by Rekaya et al. (1999), López-Romero and Carabaño (2003) and Bignardi et al. (2009) for Holstein cattle; and by Kettunen et al. (2000) for Ayrshire cattle.

When comparing the models fitting linear spline functions, a reduction in TRV, BIC, and DIC criteria was observed when additional knots were included, indicating that the knots placed at d 120 and 260 provided greater model flexibility and better fit to the data. According to the TRV values, model SPL665 provided the best fit of the data among all models tested (Table 3 ). However, the reduction in TRV was only $0.24 \%$ when compared with the second better-fit model (LEG455). The DIC and BIC criteria indicated model LEG455 to be the best model to fit the test-day records, followed by model SPL665. These results demonstrate that the residual variance should not be the unique criterion for the model comparison because it tends to select overparameterized models. One of the peculiarities of models that use test-day milk yields is the high computational requirement due to the large number of parameters to be estimated. In this respect, model SPL665 (47 parameters) results in an increase of $57 \%$ in the number of parameters to be estimated when compared with model LEG455 (30 parameters). The random-access memory (RAM) usage and the time spent per iteration were 46 and $57 \%$ higher for this model, respectively, compared with model LEG455. Another important aspect is the correlation structure between model parameters, which is related to convergence speed (Schaeffer, 2008). The PRRC was $0.9 \%$ for model SPL665 and $81.6 \%$ for model LEG455, indicating that the interdependence between parameters of model SPL665 is markedly higher than that seen for model LEG455. Similarly, Guo and Schaeffer (2002) reported higher correlations between parameters and lower PRRC values for spline functions compared with other functions.
Bohmanova et al. (2008), studying test-day data of milk, fat, and protein yield and SCC in Canadian Holstein cattle, compared 4 models in which fixed and random regressions were fitted by fourth-order Legendre polynomials (LEG4) or linear spline functions with 4, 5, or 6 knots. In contrast to the present study, the authors found that the model fitting linear splines with 6 knots (SPL6) was superior to the other models according to the DIC and TRV criteria. However, as observed in the present study, SPL6 was the most complex model and, therefore, required a longer time to reach convergence, whereas model LEG4 presented the fastest convergence time among all models tested.

The phenotypic variances obtained with models LEG455 and SPL665 showed a similar trend across lactation, with the observation of discretely higher values in the middle third of lactation (Figure 2). The estimates obtained with model LEG455 for the first days of lactation were slightly higher than those estimated with model SPL665. The environmental variances (sum of permanent environmental and residual variances) obtained with the $2 \mathrm{RRM}$ were similar, as were the genetic variances. However, as seen for phenotypic variances, the variances obtained with model LEG455 were slightly higher on the first days of lactation. However, the $95 \%$ high posterior density intervals for the variance estimates obtained with the 2 models were found to overlap across lactation.

The posterior means of heritability (Figure 3 ) showed a similar trend to that observed for genetic variances and ranged from 0.12 to 0.26 (LEG455) and from 0.16 to 0.29 (SPL665). The estimates obtained with model SPL665 were slightly higher than those obtained with model LEG455 in the first third (except between d 5 and 30) and at the end of lactation. Estimates of similar magnitude have been reported by Herrera et al. (2008) and Pereira et al. (2010) using Wilmink or Ali and Schaeffer functions to model fixed and random regressions of test-day records in dairy Gyr cattle. However, Costa et al. (2005) found much higher (around 0.80) estimates of heritability at the first $60 \mathrm{~d}$ of lactation using dairy Gyr data. Their results could be due to the small sample size used (1,200 cows).

The posterior means of phenotypic correlations ranged from 0.20 to 0.88 (LEG455) and from 0.21 to 0.87 (SPL665). Higher correlations were observed between adjacent test-day records (Figure 4). The permanent environmental correlation estimates presented a similar trend, as expected and ranged from 0.28 to 0.99 (LEG455) and from 0.29 to 0.99 (SPL665). Similarly, the genetic correlation estimates obtained with the 2 models were about 0.99 between adjacent testday records and decreased to 0.10 between test days at 

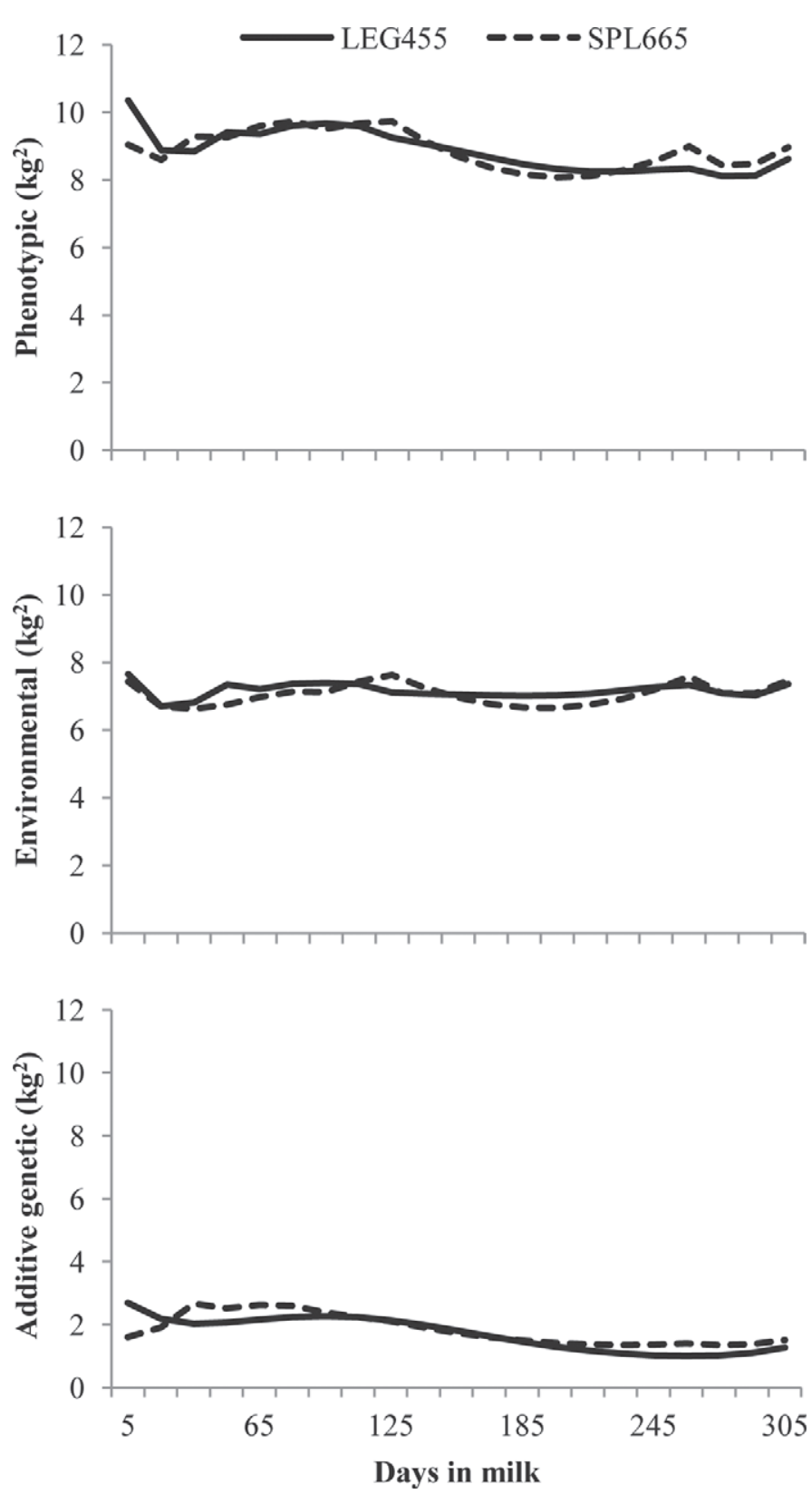

Figure 2. Posterior means estimates of phenotypic, environmental (sum of permanent environmental and residual variances), and additive genetic variances according to DIM (LEG455 = model using 3and 4-order Legendre polynomials for additive genetic and permanent environmental effects and 5 classes of residual variance; SPL665 = model using linear spline functions with 6 random regression coefficients for additive genetic and permanent environmental effects and 5 classes of residual variance).

the beginning and at the end of lactation. The genetic correlations between test-day records estimated with model SPL665 showed sudden and unexpected declines with increasing distance between days of lactation. The correlations between the fifth day of lactation and the remaining days decreased from values close to 1

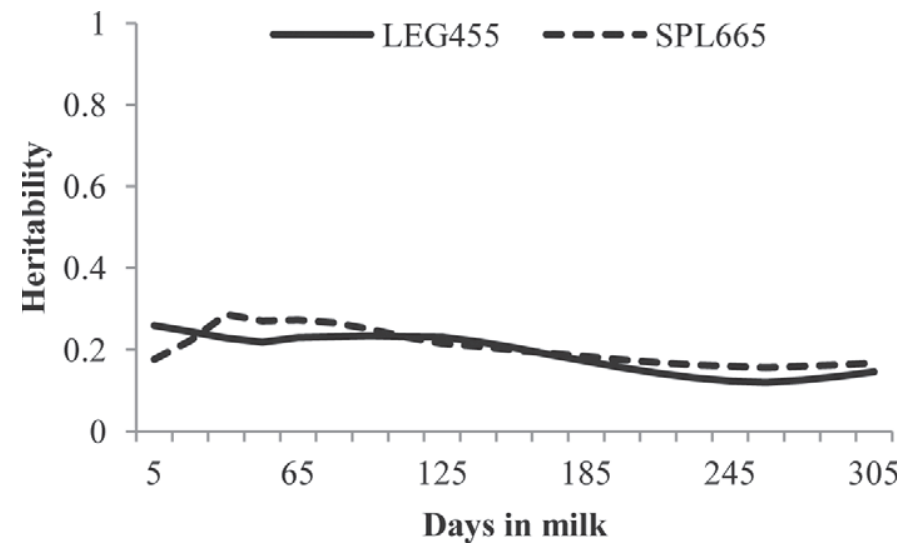

Figure 3. Posterior means estimates of heritability according to DIM (LEG455 = model using 3- and 4-order Legendre polynomials for additive genetic and permanent environmental effects and 5 classes of residual variance; SPL665 = model using linear spline functions with 6 random regression coefficients for additive genetic and permanent environmental effects and 5 classes of residual variance).

to values of less than 0.20 after $155 \mathrm{~d}$ of lactation. In addition, the $95 \%$ high posterior density intervals for genetic correlation estimates were substantially higher for model SPL665 compared with model LEG455, indicating that the model using linear spline functions provided less accurate correlation estimates. Similar results in terms of magnitude and trend of genetic correlations have been reported by Herrera et al. (2008) for dairy Gyr; however, the test-day records were grouped in ten 30-d classes in their study. In contrast, Pereira et al. (2010), who analyzed part of the same data set using Wilmink or Ali and Schaeffer functions to model fixed and random regressions, reported negative genetic correlations between test-day records at the beginning and at the end of lactation.

Given the occurrence of short lactations (less than $200 \mathrm{~d}$ ), a genetic peculiarity of this breed, there is a marked reduction in the number of observations in the last third of lactation. This could affect estimates of genetic parameters and be one of the causes of the observed decrease in heritability estimates after $180 \mathrm{~d}$ of lactation, and the low or negative genetic correlations estimated between yields at the beginning and at the end of lactation. Studies to determine whether the period of $305 \mathrm{~d}$ is the most appropriate to be used for genetic evaluations of this breed are recommended (Costa et al., 2005).

For additive genetic effects, the 3 principal eigenvalues of the covariance matrix between test-day records explained 99.3\% (LEG455) and $98.2 \%$ (SPL665) of the total genetic variation between animals. When the LEG455 model was used, each of the 3 principal eigenvalues explained $78.9,14.7$, and $5.8 \%$ of the variation, 

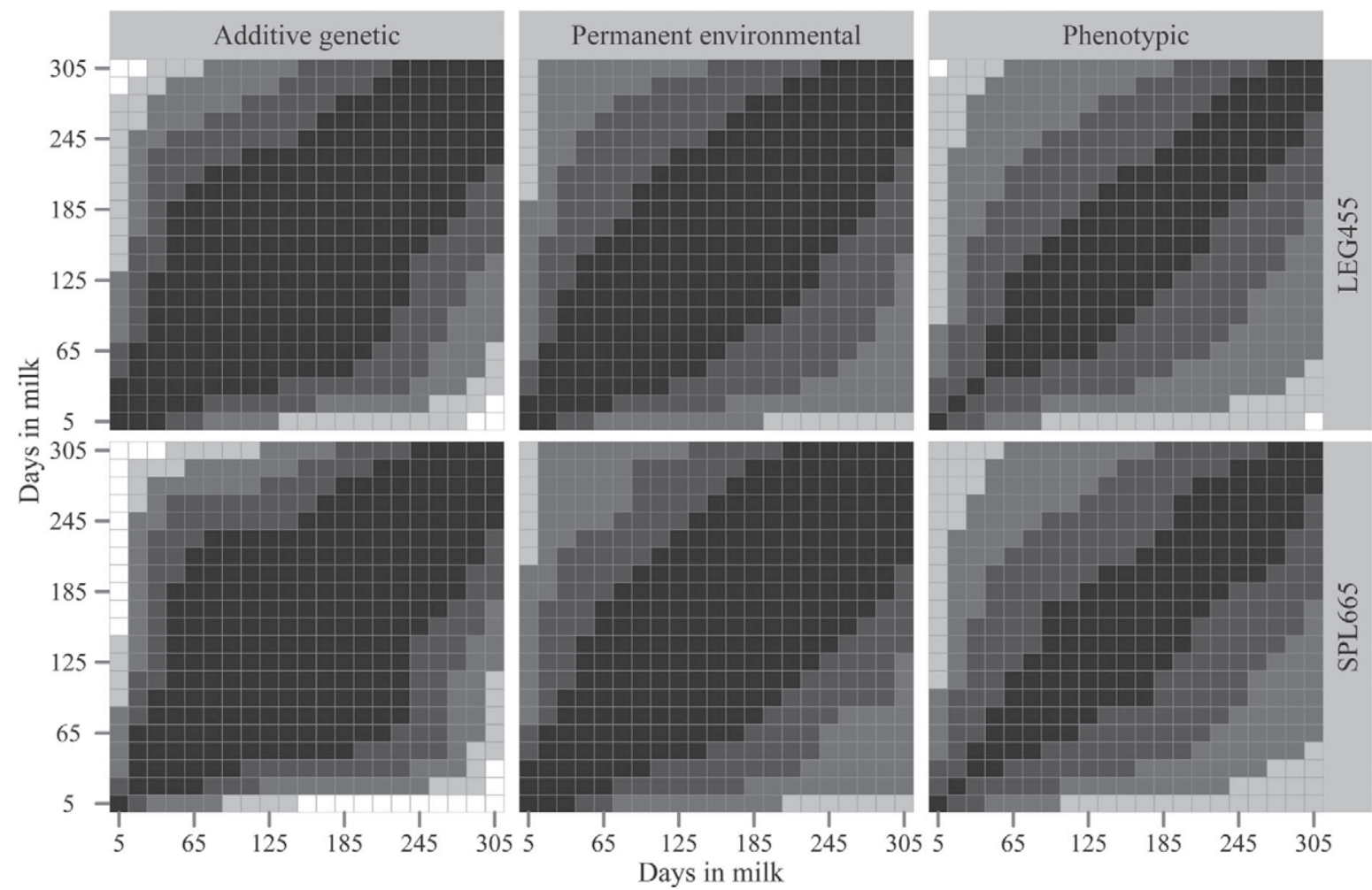

\section{Correlation}

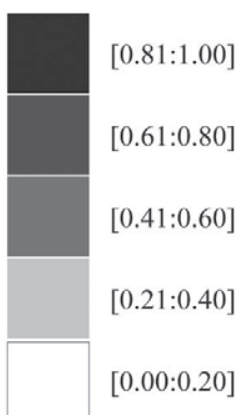

Figure 4. Posterior means estimates of additive genetic, permanent environmental, and phenotypic correlations between days of lactation (LEG455 = model using 3- and 4-order Legendre polynomials for additive genetic and permanent environmental effects and 5 classes of residual variance; SPL665 = model using linear spline functions with 6 random regression coefficients for additive genetic and permanent environmental effects and 5 classes of residual variance).

respectively. A large eigenvalue (proportional to the other eigenvalues) indicates that selection based on its corresponding eigenfunction will result in faster changes than those observed when an eigenfunction with a small eigenvalue is used (Kirkpatrick et al., 1990). Similarly, for permanent environmental effects, the 3 principal eigenvalues explained $95.9 \%$ (LEG455) and 97.2\% (SPL665) of the total genetic variation. The last eigenvalue obtained with model SPL665 for both additive genetic and permanent environmental effects was almost 0 , indicating that the 3 main eigenvalues of the covariance matrix between test-day records explained almost all the genetic variation of this trait; therefore, a possibility exists of fitting a reduced rank model with a smaller number of parameters.

The eigenfunctions corresponding to the 3 principal eigenvalues of the covariance matrix between test days for additive genetic and permanent environmental effects obtained with models LEG455 and SPL665 presented similar trends. The 3 principal eigenfunctions obtained with model LEG455 are illustrated in Figure 5. The first eigenfunction was positive and almost constant throughout lactation. The associated EBV corresponds to the average potential of the animal across lactation (Druet et al., 2003). Selection based on the first eigenfunction permits changing milk production across lactation in the same direction. In contrast, the second eigenfunction increased from the beginning $(-0.46)$ to the end of lactation (0.31), indicating contrasting relationships between EBV for milk production at the beginning and at the end of the lactation period. Selection based on this eigenfunction may allow changing the shape of the lactation curve (persistency of milk yield) of the population (Olori et al., 1999). The third eigenfunction was positive at the beginning and at the end of lactation and was negative during midlactation. Togashi and Lin $(2006,2007)$ demonstrated that the 3 principal eigenvectors of the covariance matrix between random regression coefficients for additive genetic effects can be used for the construction of a selection index to maximize milk production during lactation and persistency.

Rank correlations between bull EBV from different models ranged from 0.946 up to 0.998 (Table 4). The highest correlations were between EBV from models applying the same type of function (SPL or LEG). On the other hand, rank correlations were lower when comparing SPL and LEG models. The lowest correlation 
Table 4. Pearson (above diagonal) and Spearman rank (under diagonal) correlations between EBV for 305-d cumulative milk yield

\begin{tabular}{lccccc}
\hline & \multicolumn{5}{c}{ Correlation } \\
\cline { 2 - 6 } Model $^{1}$ & LEG445 & LEG455 & SPL445 & SPL555 & SPL665 \\
\hline LEG445 & - & 0.999 & 0.989 & 0.976 & 0.986 \\
LEG455 & 0.998 & - & 0.986 & 0.968 & 0.980 \\
SPL445 & 0.980 & 0.975 & - & 0.991 & 0.995 \\
SPL555 & 0.958 & 0.946 & 0.986 & - & 0.995 \\
SPL665 & 0.975 & 0.964 & 0.990 & 0.992 & -
\end{tabular}

${ }^{1}$ Models are presented according to the code Tkakpr, where $T$ is the type of basis function used (orthogonal Legendre polynomial, LEG, or linear spline function, SPL); $k a$ and $k p$ are the numbers of random regression coefficients for additive genetic and permanent environmental effects, respectively; and $r$ is the number of residual variance classes.

observed (0.946) was between LEG455 and SPL555, indicating significant changes in rank between these models. The high rank correlation between bull EBV obtained with LEG445 and LEG455 (0.998) indicates that virtually no differences exist between them for selecting bulls. Additionally, the genetic parameters estimated with these models were practically the same. Pearson correlations among EBV from different models were very similar to rank correlations.

Accurate estimates of genetic parameters can be obtained using random regression models; however, large data sets are required for this type of analysis (Druet et al., 2003). The dairy Gyr, can be considered a small population, but due to its desirable characteristics such as adaptation to heat, resistance to endo- and ectoparasites, and good milk production in grazing systems, it is in expansion, with an increasing number of breeders in Brazil and other countries located in tropical areas.

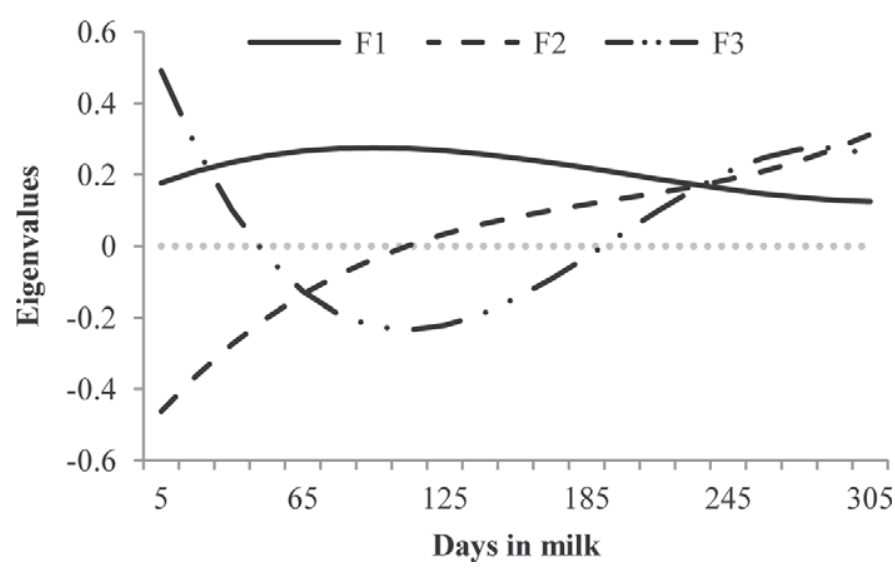

Figure 5. Posterior means estimates of the eigenfunctions corresponding to the 3 principal (F1, F2, and F3) eigenvalues of the covariance matrix between daily milk yields for additive genetic effect (LEG455 = model using 3- and 4-order Legendre polynomials for additive genetic and permanent environmental effects and 5 classes of residual variance).
International cooperation would be an opportunity to increase the dairy Gyr database.

Further studies using spline functions should consider different knot positions to better fit test-day records of milk. In addition, an increase in knot density should be evaluated. However, an increased knot density may substantially raise the number of parameters estimated and possibly reduce the accuracy of the genetic parameter estimates. Another possibility is the investigation of different types of functions for fixed and random regressions (e.g., Legendre polynomials for additive genetic effects and linear spline functions for permanent environmental effects), but large changes in overall model performance are not expected.

\section{CONCLUSIONS}

Results from the current study showed that heritability estimates obtained with Legendre polynomials and splines models were similar; however, negative genetic correlations between test-day yields at the beginning and at the end of lactation were estimated with all linear spline models, which are not biologically expected. Consequently, Legendre polynomial random regression models are indicated for fitting test-day milk yields in the dairy Gyr breed. A model using cubic Legendre polynomials for additive genetic and permanent environmental effects and 5 classes of residual variance is a parsimonious option that fits test-day milk yield adequately. The next step will be to study whether this model could be used in genetic evaluations of the dairy Gyr breed for milk yield, with the objective of obtaining more accurate EBV when compared with those obtained with the current model, which is based on lactation model.

\section{ACKNOWLEDGMENTS}

This work was funded by Fundação de Amparo à Pesquisa do Estado de São Paulo (FAPESP), Brazil. 
The suggestions and comments made by editor and reviewers are acknowledged.

\section{REFERENCES}

Bignardi, A. B., L. El Faro, V. L. Cardoso, P. F. Machado, and L. G. de Albuquerque. 2009. Random regression models to estimate testday milk yield genetic parameters Holstein cows in Southeastern Brazil. Livest. Sci. 123:1-7.

Bohmanova, J., F. Miglior, J. Jamrozik, I. Misztal, and P. G. Sullivan. 2008. Comparison of random regression models with Legendre polynomials and linear splines for production traits and somatic cell score of Canadian Holstein cows. J. Dairy Sci. 91:3627-3638.

Bozdogan, H. 2000. Akaike's information criterion and recent developments in information complexity. J. Math. Psychol. 44:62-91.

Costa, C. N., C. M. R. de Melo, C. H. C. Machado, A. F. de Freitas, I. U. Packer, and J. de Araújo Cobuci. 2005. Parâmetros genéticos para a produção de leite de controles individuais de vacas da raça Gir estimados com modelos de repetibilidade e regressão aleatória. R. Bras. Zootec. 34:1519-1530.

Druet, T., F. Jaffrézic, D. Boichard, and V. Ducrocq. 2003. Modeling lactation curves and estimation of genetic parameters for first lactation test-day records of French Holstein cows. J. Dairy Sci. 86:2480-2490.

El Faro, L., V. L. Cardoso, and L. G. de Albuquerque. 2008. Variance component estimates for test-day milk yield applying random regression models. Genet. Mol. Biol. 31:665-673.

Geweke, J. 1992. Evaluating the accuracy of sampling-based approaches to the calculation of posterior moments (with discussion). Pages 169-193 in Bayesian Statistics. Vol. 4. J. M. Bernardo, J. O. Berger, A. P. Dawid, and A. F. Smith, ed. Oxford University Press, Oxford, UK.

Guo, Z., and L. R. Schaeffer. 2002. Random regression submodel comparison. 7th World Congr. Genet. Appl. Livest. Prod. Montpellier, France. CD-ROM Commun. No. 20-08.

Heidelberger, P., and P. Welch. 1983. Simulation run length control in the presence of an initial transient. Oper. Res. 31:1109-1144.

Herrera, L. G. G., L. El Faro, L. G. de Albuquerque, H. Tonhati, and C. H. C. Machado. 2008. Estimativas de parâmetros genéticos para a produção de leite e persistência da lactação em vacas Gir aplicando modelos de regressão aleatória. R. Bras. Zootec. 37:1584-1594.

Interbull. 2012. Description of National Genetic Evaluation Systems for dairy cattle traits as applied in different Interbull member countries. Accessed Aug. 11, 2012. http://www-interbull.slu.se/ national_ges_info2/framesida-ges.htm.

Kettunen, A., E. A. Mäntysaari, and J. Pösö. 2000. Estimation of genetic parameters daily milk yield of primiparous Ayrshire cows by random regression test-day models. Livest. Prod. Sci. 66:251-261.

Kirkpatrick, M., D. Lofsvold, and M. Bulmer. 1990. Analysis of the inheritance, selection and evolution of growth trajectories. Genetics 124:979-993.

López-Romero, P., and M. J. Carabaño. 2003. Comparing alternative random regression models to analyse first lactation daily milk yield data in Holstein-Friesian cattle. Livest. Prod. Sci. 82:81-96.
Meyer, K. 1999. Estimates of genetic and phenotypic covariance functions for postweaning growth and mature weight of beef cows. J. Anim. Breed. Genet. 116:181-205.

Meyer, K. 2005. Random regression analyses using B-splines to model growth of Australian Angus cattle. Genet. Sel. Evol. 37:473-500.

Misztal, I. 2006. Properties of random regression models using linear splines. J. Anim. Breed. Genet. 123:74-80.

Misztal, I. 2010. BLUPF90 Manual. Accessed Dec. 10, 2010. http:// nce.ads.uga.edu/ ignacy/newprograms.html.

Olori, V. E., W. G. Hill, B. J. McGuirk, and S. Brotherstone. 1999. Estimating variance components for test day milk records by restricted maximum likelihood with a random regression animal model. Livest. Prod. Sci. 61:53-63.

Pereira, R. J., P. S. Lopes, R. da Silva Verneque, M. L. Santana Júnior, M. R. Lagrotta, and R. de Almeida Torres. 2010. Funções de covariância para produção de leite no dia do controle em bovinos Gir leiteiro. Pesquisa Agropecu. Bras. 45:1303-1311.

Reis Filho, J. C. 2006. Endogamia na raça Gir. MS Thesis. Universidade Federal de Viçosa, Minas Gerais, Brazil.

Rekaya, R., M. J. Carabaño, and M. A. Toro. 1999. Use of test-day yield for the genetic evaluation of production traits in HolsteinFriesian cattle. Livest. Prod. Sci. 57:203-217.

Rice, J. A., and C. O. Wu. 2001. Nonparametric mixed effects models for unequally sampled noisy curves . Biometrics 57:253-259.

Schaeffer, L. R. 2008. Random regression models. Accessed Jun. 05, 2008. http://www.aps.uoguelph.ca/\%7Elrs/ABModels/NOTES/ RRM14a.pdf.

Schaeffer, L. R., J. Jamrozik, G. J. Kistemaker, and B. J. Van Doormaal. 2000. Experience with a test-day model. J. Dairy Sci. $83: 1135-1144$.

Schwarz, G. 1978. Estimating the dimension of a model. Ann. Stat. 6:461-464

Smith, B. J. 2007. boa: An R Package for MCMC Output Convergence Assessment and Posterior Inference. J. Stat. Softw. 21:1-37.

Spiegelhalter, D. J., N. G. Best, B. P. Carlin, and A. van der Linde. 2002. Bayesian measures of model complexity and fit. J. R. Stat. Soc. Ser. B (Stat. Methodol.) 64:583-639.

Swalve, H. H. 2000. Theoretical basis and computational methods for different test-day genetic evaluation methods. J. Dairy Sci. 83:1115-1124.

Togashi, K., and C. Y. Lin. 2006. Selection for milk production and persistency using eigenvectors of the random regression coefficient matrix. J. Dairy Sci. 89:4866-4873.

Togashi, K., and C. Y. Lin. 2007. Genetic modification of the lactation curve by bending the eigenvectors of the additive genetic random regression coefficient matrix. J. Dairy Sci. 90:5753-5758.

Verneque, R. S., J. C. C. Panetto, F. A. T. Bruneli, C. C. Geraldo, M. A. Machado, A. R. Fernandes, R. S. Maciel, C. H. C. Machado, A. E. Vercesi Filho, M. V. G. B. Silva, G. G. Santos, and M. G. C. D. Peixoto. 2011. Programa Nacional de Melhoramento do Gir Leiteiro-Sumário Brasileiro de Touros-Resultado do Teste de Progênie-Maio 2011 (Documentos, 145). Embrapa Gado de Leite, Juiz de Fora, Minas Gerais, Brazil. 